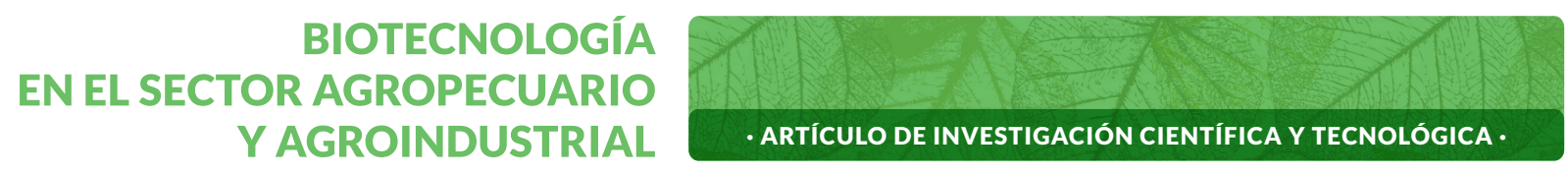

\title{
Propiedades fisicoquímicas y funcionales del almidón obtenido de dos variedades de batata (Ipomoea batatas)*
}

\section{Physicochemical and functional properties of almidon obtained from two varieties of sweet potatoes (Ipomoea batatas)}

\section{Propriedades físico-químicas e funcionais do almidon obtido de duas variedades de batata (Ipomoea batatas)}

MURILLO-MARTÍNEZ, MARÍA ${ }^{1}$; ALVIS-BERMÚDEZ, ARMANDO²;

ARRAZOLA-PATERNINA, GUILLERMO ${ }^{3}$

Historial del Artículo

Recibido para evaluación: 6 de Marzo 2020.

Aprobado para publicación: 20 de Octubre 2020.

* Titulo del proyecto de origen: "Aprovechamiento agroindustrial de dos variedades de batata (Ipomoea batatas)". Financiación: Universidad de Córdoba, Convocatoria interna. Culminación: Junio 30 de 2018.

1 Universidad de Córdoba, Facultad de Ingenierías, Programa de Ingeniería de Alimentos, Grupo de Investigación Procesos y Agroindustria de vegetales. Ingeniero de Alimentos. Montería, Colombia. https://orcid.org/0000-0001-7071-9107

2 Universidad de Córdoba, Facultad de Ingenierías, Programa de Ingeniería de Alimentos, Grupo de Investigación Procesos y Agroindustria de vegetales. PhD. Ingeniería. Montería, Colombia. https://orcid.org/0000-0002-5638-271X

3 Universidad de Córdoba, Facultad de Ingenierías, Programa de Ingeniería de Alimentos, Grupo de Investigación Procesos y Agroindustria de vegetales. PhD. Ingeniería. Montería, Colombia. https://orcid.org/0000-0001-6878-0806

Correspondencia: gsarrazola@correo.unicordoba.edu.co 


\section{RESUMEN}

El cultivo y producción de la Batata en Colombia registra pocos excedentes para el mercado nacional, su rendimiento se encuentra aproximadamente en 6 t/ha, dicha producción, se centra en la región Caribe Colombiana, en los departamentos de Córdoba y Sucre; sin embargo, el cultivo de la batata podría llegar a ocupar un lugar de importancia para la industria alimentaria, por los aportes nutricionales y por su alto contenido de almidón, considerado ideal para la obtención industrial de harinas y almidones. Esta investigación estudio las propiedades fisicoquímicas y funcionales al almidón obtenido de dos variedades de batata, donde, se analizó la capacidad de absorción de agua, viscosidad máxima, temperatura de gelatinización, asentamiento, estabilidad. Previo a las determinaciones, las muestras fueron secadas hasta peso constante. Se encontró que el almidón de batata amarilla resulto ser de mejor calidad al poseer menor solubilidad, mayor absorción de agua, mayor poder de hinchamiento y mayor temperatura de gelatinización que el almidón de batata morada, mostrando valores alentadores para la industria alimentaria como espesantes, estabilizantes y gelificantes en alimentos refrigerados y congelados.

\section{ABSTRACT}

Sweet potato cultivation and production in Colombia registers few surpluses for the national market, its yield is approximately $6 \mathrm{t} / \mathrm{ha}$, said production is centered in the Colombian Caribbean region, in the departments of Córdoba and Sucre; However, the cultivation of sweet potatoes could come to occupy an important place for the food industry, due to its nutritional contributions and its high starch content, considered ideal for the industrial production of flours and starches. This investigation studied the physicochemical and functional properties of the starch obtained from two varieties of sweet potato, where the water absorption capacity, maximum viscosity, gelatinization temperature, settling, stability was analyzed. Before the determinations, the samples were dried to constant weight. It was found that the yellow sweet potato starch turned out to be of better quality due to its lower solubility, higher water absorption, higher swelling power and higher gelatinization temperature than the purple sweet potato starch, showing encouraging values for the food industry as thickeners, stabilizers and gelling agents in refrigerated and frozen foods.

\section{RESUMO}

O cultivo e a produção de batata-doce na Colômbia registram poucos excedentes para o mercado nacional, sua produção é de aproximadamente $6 \mathrm{t} / \mathrm{h}$, disse que a produção está centrada na região do Caribe colombiano, nos departamentos de Córdoba e Sucre; No entanto, o cultivo da batata-doce pode

Cómo citar este artículo: MURILLO-MARTÍNEZ, MARÍA; ALVIS-BERMÚDEZ, ARMANDO; ARRAZOLA-PATERNINA, GUILLERMO. Propiedades fisicoquímicas y funcionales del almidón obtenido de dos variedades de batata (Ipomoea batatas). Biotecnología en el sector agropecuario y agroindustrial, v. 19, n. 1, 2021, p. xx-xx. Doi:

\section{PALABRAS CLAVE:} Reología; Gelatinización; Absorción; Hinchamiento; Tecnofuncionales.

\section{KEYWORDS:}

Rheology; Gelatinization; Absorption; Swelling;

Techofunctional.

\section{PALAVRAS-CHAVE:}

Reologia; Gelatinização;

Absorção; Inchaço;

Tecofuncionais. 
vir a ocupar um lugar importante para a indústria alimentícia, devido às suas contribuições nutricionais e ao seu alto teor de amido, considerado ideal para a produção industrial de farinhas e amidos. Esta investigação estudou as propriedades físico-químicas e funcionais do amido obtidas de duas variedades de batata-doce, onde foram analisadas a capacidade de absorção de água, viscosidade máxima, temperatura de gelatinização, sedimentação e estabilidade. Antes das determinações, as amostras foram secas com peso constante. Verificou-se que o amido de batata-doce amarelo resultou em melhor qualidade devido à sua menor solubilidade, maior absorção de água, maior poder de intumescimento e maior temperatura de gelatinização que o amido de batata-doce roxo, mostrando valores encorajadores para a indústria de alimentos como espessantes, estabilizadores e gelificantes em alimentos refrigerados e congelados.

\section{INTRODUCCIÓN}

La batata (Ipomea batatas Lam.) pertenece al grupo de raíces y tubérculos, conocidos por ser fuentes principales de carbohidratos de gran aporte calórico (almidón), sin dejar de lado la presencia en su composición de otros nutrientes [1] Las características de la batata como cultivo resistente, la convierten en un actor principal de la seguridad alimentaria, en cuanto a disponibilidad, accesibilidad, estabilidad y utilización. Alrededor de 105 millones de toneladas de este alimento se produjeron en el mundo en 2016 [2].

En la actualidad existen muchas variedades de batata, las cuales se diferencian dependiendo de sus atributos físicos y químicos, un ejemplo de esto es la gran cantidad de betacaroteno presente en la batata de pulpa naranja o amarilla, este atributo ha ocasionado un aumento en la demanda de este tipo de batata, particularmente en la industria de alimentos donde se utiliza como ingrediente para productos de panadería, aperitivos, espesantes y de confitería el cual ha llevado a una creciente demanda e interés por este tipo de batata [3] A su vez puede transformarse en harina para una vida útil más larga [4].

Otro componente importante que posee la batata es el almidón, fuente de energía importante en la dieta humana. Con el inicio de la industria de alimentos procesados se dio paso a la necesidad de disponibilidad de almidón puro y por ende al crecimiento en el uso de estos [5]. La utilidad del almidón en formulaciones y aplicaciones industriales depende de las siguientes propiedades: gelatinización, retrogradación; solubilidad, hinchamiento, absorción de agua, sinéresis y comportamiento reológico de sus pastas y geles, por lo anterior se hace necesario conocer las propiedades funcionales, debido a que las investigaciones referentes a las raíces como camote, ñampí (Malanga), ñame y yuca aún se encuentran en etapa inicial [6].

El objetivo del presente trabajo fue evaluar las propiedades fisicoquímicas y funcionales del almidón obtenido a partir de las variedades de batata morada pulpa blanca y amarilla pulpa naranja (Ipomoea batatas).

\section{MÉTODO}

\section{Recolección de las muestras}

$10 \mathrm{~kg}$ de batatas fueron obtenidas en el mercado público del sur de Montería (Córdoba- Colombia), 5 kg de batata amarilla y $5 \mathrm{~kg}$ de batata morada, localizado geográficamente en la coordenada, $8^{\circ} 44^{\prime} 34^{\prime \prime}$ Norte y $75^{\circ}$ $53^{\prime} 14$ " Oeste a $20 \mathrm{msnm}$, temperatura promedio de $28^{\circ} \mathrm{C}$ y humedad relativa de $85 \%$, libres de daños mecánicos y de microorganismos, las batatas fueron suministradas por un mismo proveedor, con el objetivo de minimizar la posibilidad de mezclas varietales, siendo escogidas las raíces de batata tipo morada con la mayor homogeneidad posible en tamaño, madurez y peso. 


\section{Extracción de almidón de batata}

El almidón se obtuvo siguiendo la metodología propuesta por [7] en la cual las muestras de las dos variedades de batata (amarilla y morada) fueron peladas, cortadas en cubos de aproximadamente $3 \mathrm{~cm}$ y sumergidos por 30 minutos en solución de Bisulfito de Sodio $\left(\mathrm{NaHSO}_{3}\right)(1500 \mathrm{ppm}, 1: 3(\mathrm{p} / \mathrm{v}))$. Posteriormente los cubos fueron triturados durante 2 minutos en una licuadora industrial y la masa resultante se mezcló en recipientes que contenían solución de $\mathrm{NaHSO}_{3}\left(1500 \mathrm{ppm}\right.$ de $\left.\mathrm{SO}_{2}, 1: 1(\mathrm{v} / \mathrm{v})\right)$. Seguidamente la lechada de almidón se filtró para eliminar la fibra y se dejó en reposo para sedimentación a $4^{\circ} \mathrm{C}$ por 4 horas. Luego el líquido sobrenadante se eliminó y el sedimento se lavó 3 veces con agua destilada, centrifugando en el último lavado a 2500 rpm, por 12 minutos con la finalidad de recuperar el almidón. Posteriormente, se secó en una estufa a $55^{\circ} \mathrm{C}$, durante 24 horas para posterior molienda en el equipo Pulverisette 16 hasta que se obtuvo un polvo (malla 100), luego se almacenó en frascos de vidrio con cierre de tapa hermética hasta su uso.

\section{Caracterización fisicoquímica del almidón}

Se evaluó la composición fisicoquímica del almidón obtenido a partir de las variedades de batata morada y amarilla de acuerdo a la metodología descrita por [8]. Se evaluaron las siguientes propiedades: humedad (AOAC 925.10), cenizas (AOAC 923.03), grasa bruta (AOAC 920.85), proteína bruta (AOAC 920.87), y fibra bruta (AOAC 985.29).

Los carbohidratos totales fueron obtenidos mediante diferencia de los datos reportados en el análisis proximal, tal como se muestra en la Ecuación 1.

$$
\text { (\%)CHOS }=100-(\%) H u m .-(\%) \text { Pro. }-(\%) \text { Gra. }-(\%) \text { Fib. }-(\%) \text { Cen. }
$$

La energía se calculó utilizando los factores de conversión de Atwater, como se muestra en la Ecuación 2.

$$
\text { Calorías }\left(\frac{\text { Kcal }}{100 g}\right)=(4 * \% \text { pro. })+(9 * \% \text { gra. })+(4 * \% \text { chos })
$$

\section{Caracterización funcional del almidón}

Se ejecutaron los siguientes ensayos: Índice de absorción de agua (IAA), índice de solubilidad en agua (ISA) y poder de hinchamiento (PH): utilizando la técnica escrita por [9] la cual consistió en agregar 1,25 g de la muestra de almidón de batata a los tubos de centrifuga seco, más $30 \mathrm{~mL}$ de agua destilada precalentada a $60^{\circ} \mathrm{C}$, se procedió a agitación y después se llevaron los tubos a baño maría por 30 minutos a $60^{\circ} \mathrm{C}$, la agitación de la suspensión se comenzó a los 10 minutos de iniciado el calentamiento. Seguidamente se centrifugaron a 4900 RPM por 30 minutos a temperatura ambiente, se separó el sobrenadante y midió el volumen en una probeta graduada, luego se tomaron $10 \mathrm{~mL}$ del sobrenadante, se adicionaron en un vaso de precipitado y se secó el sobrenadante en un horno a $70^{\circ} \mathrm{C}$. Finalmente, se pesó el tubo de centrifuga con el gel y el vaso de precipitado con los insolubles en una balanza analítica. Para la determinación del IAA, ISA y PH se utilizaron las ecuaciones 3, 4 y 5.

$$
\begin{aligned}
I A A & =\frac{\text { Peso del gel }(g)}{\text { Peso muestra }(g)} \\
I S A & =\frac{\text { Peso solubles }(g) \times V \times 10}{\text { Peso muestra }(g)}
\end{aligned}
$$




$$
\left.P H=\frac{\text { Peso del gel }(g)}{\text { Peso muestra }(g)-\text { Peso solubles }(g)} \quad \text { (Ec. } 5\right)
$$

\section{Temperatura de gelatinización}

Se utilizó la técnica descrito por [10], la cual consistió en adicionar $10 \mathrm{~g}$ de almidón de batata en un frasco volumétrico, disolver y completar con agua destilada hasta $100 \mathrm{~mL}$, seguidamente se tomaron $50 \mathrm{~mL}$ de la suspensión de almidón de batata y se agregaron a un vaso de precipitado de $100 \mathrm{~mL}$, se calentó en baño maría a $85^{\circ} \mathrm{C}$ y se agitó de manera constante la suspensión de almidón hasta la formación de una pasta en conjunto con una temperatura estable. La temperatura de gelatinización se determinó directamente del termómetro.

Se utilizó un análisis de varianza (ANOVA) con un nivel de confianza del 95\%, al momento de encontrar diferencias significativas $(p<0,05)$ se compararon mediante una prueba t-student.

\section{RESULTADOS}

\section{Caracterización fisicoquímica del almidón de batata morada y amarilla.}

Los resultados obtenidos de la caracterización fisicoquímica del almidón de batata morada y amarilla se muestran en el Cuadro 1.

\section{Análisis de Humedad}

Los valores obtenidos de la humedad del almidón de batata morada y de batata amarilla fueron 10,95\% y $10,91 \%$ respectivamente, valores muy cercanos a otros reportados en $5,50-19,14 \%$ [11], pero superiores a $3,86-6,52 \%[7], 7,34 \%[12], 8,03 \%[1]$ y $9,83 \%$ [13].

Esta diferencia de resultados puede obedecer a que la humedad depende del método utilizado y de las condiciones de deshidratación utilizadas [14]. Valores de humedad superiores al 13\% puede ocasionar daño microbiano y por lo tanto, el deterioro en la calidad del polímero [12].

Para porcentaje de humedad, se describen valores para otros tipos de tubérculos y raíces como almidón de ñame (8,98\%), almidón de yuca (9,12\%) reportados por [1]; almidón de ñame (8,66-10,22\%) y almidón de yuca

Cuadro 1. Composición fisicoquímica del almidón de batata*

\begin{tabular}{|c|c|c|}
\hline Variables & Almidón batata morada & Almidón batata amarilla \\
\hline Humedad (\%) & $10,95 \pm 0,49^{\mathrm{a}}$ & $10,91 \pm 0,13^{\mathrm{a}}$ \\
\hline Cenizas (\%) & $0,32 \pm 0,02^{\mathrm{a}}$ & $0,38 \pm 0,05^{\mathrm{a}}$ \\
\hline Fibra cruda (\%) & $0,43 \pm 0,01^{\mathrm{a}}$ & $0,47 \pm 0,04^{\mathrm{a}}$ \\
\hline Proteína (\%) & $0,38 \pm 0,04^{\mathrm{a}}$ & $0,34 \pm 0,08^{\mathrm{a}}$ \\
\hline Grasa (\%) & $0,46 \pm 0,29^{\mathrm{a}}$ & $0,20 \pm 0,10^{\mathrm{a}}$ \\
\hline Carbohidratos totales (\%) & $87,35 \pm 0,69^{\mathrm{a}}$ & $87,69 \pm 0,18^{\mathrm{a}}$ \\
\hline Energía (Kcal/100 g almidón) & $355,99 \pm 5,34^{\mathrm{a}}$ & $353,91 \pm 1,34^{\text {a }}$ \\
\hline *Letras iguales en la misma fila no denotan diferencia estadística significativa (P>0,05). \\
\hline
\end{tabular}


(7,80-8,47\%) [15]; almidón de yuca (9,48\%) y almidón de papa (19\%) [7]. Como se observa en el Cuadro 1, los porcentajes de humedad del almidón de batata morada y amarilla obtenidos son superiores a los porcentajes del almidón de ñame y yuca e inferiores al almidón de papa.

\section{Análisis de Cenizas}

Los reportes del contenido de cenizas del almidón de batata morada y amarilla fueron 0,32 y 0,38\% respectivamente, demostrando que son acordes con los reportados por otros autores, para almidón de batata (0,10$0,47 \%)$ [7], (0,31\%) [12] y $(0,26 \%)[13]$.

El porcentaje de cenizas reportado en la literatura para otros tipos de tubérculos y raíces como, el almidón de ñame $(0,40 \%)$, almidón de yuca $(2,1 \%)$, almidón de papa $(0,40 \%)$ reportado por [16]; almidón de papa $(0,27-$ $0,86 \%)$ obtenido por [7]; almidón de ñame $(0,36 \%)$, almidón de yuca $(0,34 \%)$ y almidón de papa $(0,44 \%)$ reportados por [15], valores cercanos a los obtenidos para almidón de batata en esta investigación.

\section{Análisis de Fibra Cruda}

En el cuadro 1 se presentan los resultados del porcentaje de fibra cruda del almidón de batata morada y amarilla, cuyos valores fueron de 0,43 y $0,47 \%$ respectivamente, estos valores son superiores al reportado por [7] $(0,28 \%)$. Además, otros autores muestran para fibra cruda estudios para otros tipos de tubérculos y raíces, para almidón de yuca (0,05\%) y almidón de papa $(0,05 \%$ ) [15] y almidón de ñame $(0,22 \%)$ [17], valores por debajo de los reportados para almidón de batata en esta investigación. El \% de fibra es importante ya que ayuda a mantener el corazón sano y ayuda a la liberación lenta y sostenida de la energía, lo cual ayuda a mantener estable los niveles de azúcar en sangre a lo largo del día y al control del estreñimiento.

\section{Análisis de Proteína}

Los reportes del contenido de proteína del almidón de batata morada y amarilla fueron 0,38 y 0,34\% respectivamente demostrando que están acordes con los reportados por otros autores para almidón de batata, $(0,28$ $0,75 \%$ ) [12] y cercanos a 0,37\% [13] y superior a 0,22\% [7] quienes afirman que el contenido de proteína es determinante en las características estructurales y funcionales de los almidones, condicionando el uso de estos en la industria alimentaria.

El porcentaje de proteína reportado en la literatura para otro tipo de tubérculos y raíces como, almidón de ñame es de 0,86\% [1], almidón de ñame 0,80\% [18], almidón de yuca 0,60\% y almidón de papa (0,62 \%) [15], valores superiores a los que se encontraron para almidón de batata en esta investigación.

\section{Análisis de Grasa}

En el cuadro 1 se presentan los resultados del porcentaje de grasa del almidón de batata morada y amarilla, cuyos valores fueron de 0,46 y $0,20 \%$ respectivamente, estos valores son superiores al reportado por [7] (0,28\%). Los datos del contenido de grasa del almidón de batata morada y amarilla, son inferiores a otros reportados 0,52\% [1].

El contenido de grasas reportado en literatura para otros tipos de tubérculos y raíces, para almidón de yuca $(0,12 \%)$ y almidón de ñame (0,22\%) [1]; almidón de yuca (0,30-0,32\%) y almidón de papa (0,35 \%) [15] y almidón de yuca $(0,20 \%)$ y almidón de papa (0,05 \%) [7], valores inferiores al obtenido en esta investigación para almidón de batata morada, pero similares con respecto al obtenido para almidón de batata amarilla. 


\section{Análisis de Carbohidratos Totales}

En cuanto al contenido de carbohidratos totales del almidón de batata morada y amarilla fueron de 87,35 y $87,69 \%$, los cuales concuerdan con los reportados para almidón de batata con 80-90\% [19], 90\% [20], 98,11\%) [14] y 90,4\% [21].

El contenido de carbohidratos totales reportado en literatura para otros tipos de tubérculos y raíces, son $81 \%$ para almidón de ñame [18], 66,7\% para almidón de papa, 72,8\% para almidón de ñame y 86,9\% para almidón de yuca [1], valores inferiores a los obtenidos para almidón de batata en esta investigación.

\section{Análisis de Calorías}

En el cuadro 1 se presentan los resultados del porcentaje de calorías del almidón de batata morada y amarilla, cuyos valores fueron de 355,99 y $353,91 \mathrm{kcal} / 100 \mathrm{~g}$ respectivamente, concuerdan con los $351 \mathrm{Kcal} / 100 \mathrm{~g}$ almidón de batata [1]; estos autores también reportaron valores de aporte calórico de $318 \mathrm{Kcal} / 100 \mathrm{~g}$ en almidón de ñame, $364 \mathrm{Kcal} / 100 \mathrm{~g}$ en almidón de yuca y $316 \mathrm{Kcal} / 100 \mathrm{~g}$ en almidón de papa, observando así que los resultados reportados en literatura para almidón de ñame y papa son inferiores a los obtenidos para almidón de batata, por otro lado el almidón de yuca reporta un aporte calórico mayor al del almidón de batata obtenido en el presente estudio.

Las calorías permiten que el organismo desarrolle funciones elementales como digerir los alimentos y mantener la temperatura corporal. Asimismo, entregan compuestos esenciales para el funcionamiento adecuado de los órganos y del sistema inmune.

\section{Caracterización funcional del almidón de batata morada y amarilla}

En el Cuadro 2 se relacionan los resultados obtenidos de la caracterización funcional del almidón de batata morada y amarilla.

\section{Índice de absorción de agua (IAA) solubilidad en agua (ISA) y Poder de hinchamiento (PH)}

Se tiene que el índice de absorción de agua (IAA) del almidón de batata morada y amarilla tuvieron valores de 1,91 y $1,72 \mathrm{~g}_{\mathrm{gel}} / \mathrm{g}$, valores superiores a los reportados con 0,45 y $1,15 \mathrm{~g}_{\mathrm{gel}} / \mathrm{g}$ almidón de batata [11]. La cantidad de agua retenida en los gránulos de almidón está relacionado con el contenido de proteína y carbohidratos, estos autores reportaron valores entre 2,95 a 7,20 $\mathrm{g}_{\mathrm{gel}} / \mathrm{g}$ almidón de batata, valores superiores a los obtenidos en el presente estudio [20]. Con respecto a la literatura para otros tipos de tubérculos y raíces, en almidón de ñame con 2,65 g gel $/ \mathrm{g}$ almidón [18], en almidón de papa 5,83 $\mathrm{g}_{\text {gel }} / \mathrm{g}$ almidón [15] y $\mathrm{n}$ almidón de yuca 0,82-15,52\% [22], valores superiores a los obtenidos para almidón de batata en esta investigación.

Con relación al índice de solubilidad en agua (ISA) del almidón de batata morada y amarilla cuyos valores fueron de 2,91 y $0,92 \%$ respectivamente, son inferiores a los reportados por (7-16\% en almidón de batata); (8,56 a 19,97\%). La solubilidad del almidón dependerá de una serie de factores tales como la fuente de extracción [23], las fuerzas inter-asociativas entre sus moléculas, el poder de hinchamiento y la presencia de otros componentes. Se reporta

Cuadro 2. Composición funcional del almidón de batata*

\begin{tabular}{|l|c|c|}
\hline \multicolumn{1}{|c|}{ Variables } & Almidón batata morada & Almidón batata amarilla \\
\hline Índice de absorción de agua (IAA) (g agua/g almidón) & $1,91 \pm 0,28^{\mathrm{a}}$ & $1,72 \pm 0,02^{\mathrm{a}}$ \\
\hline Índice de solubilidad en agua (ISA) (\%) & $2,91 \pm 0,74^{\mathrm{a}}$ & $0,92 \pm 0,45^{\mathrm{b}}$ \\
\hline Poder de hinchamiento (PH) (\%) & $1,93 \pm 0,29^{\mathrm{a}}$ & $1,73 \pm 0,02^{\mathrm{a}}$ \\
\hline Temperatura de Gelatinización ( $\left.{ }^{\circ} \mathrm{C}\right)$ & $76,67 \pm 2,31^{\mathrm{a}}$ & $7,21^{\mathrm{a}}$ \\
\hline * Letras diferentes en la misma fila denotan diferencia estadística significativa (P<0,05). \\
\hline
\end{tabular}


para otras raíces y tubérculos como almidón de yuca 0,27-12,32\% [22], almidón de papa 4,8-7,5\% [25] y almidón de ñame 0,59-1,92\% [17], valores cercanos a los obtenidos para almidón de batata en esta investigación.

Respecto al poder de hinchamiento, los valores obtenidos con $(\mathrm{PH})$ del almidón de batata morada y amarilla presentaron valores de 1,93 y 1,73\%, son inferiores a los reportados para almidón de batata (8,79-9,11\%) [11]. Las diferencias en el poder de hinchamiento de los almidones de batata podrían atribuirse a las variaciones en las fuerzas de unión asociativa dentro de los gránulos de almidón. Con respecto a lo reportado en literatura para otros tipos de tubérculos y raíces, se tiene para almidón de papa criolla 11,4\% , almidón de ñame 2,66\% [18] y almidón de yuca 0,79-15,45\% [22], valores superiores a los obtenidos para almidón de batata en esta investigación. Una baja solubilidad, alta absorción de agua y un alto poder de hinchamiento son almidones de buena calidad y los almidones de batata, posee estas características.

\section{Temperatura de gelatinización}

Los resultados obtenidos de la temperatura de gelatinización del almidón de batata morada y amarilla (Cuadro 2) cuyos valores son 76,67 y $76,33^{\circ} \mathrm{C}$, son cercanos a otros reportados por Abegunde et al; acosta y Blanco, Velásquez y Quintero quienes reportaron valores de $62,54-75,9^{\circ} \mathrm{C}[12], 78,50^{\circ} \mathrm{C}$ [1] y mayor de $61,3^{\circ} \mathrm{C}[7]$.

Autores como Aristizabal et al.; Cerón-Lasso et al.; Salcedo et al.; muestran que la temperatura de gelatinización del almidón de yuca se encuentra en el rango $57,5-70^{\circ} \mathrm{C}$ [22], para almidón de papa $62^{\circ} \mathrm{C}$ [26] siendo inferiores a los valores obtenidos en esta investigación y almidón de ñame $83-84,2^{\circ} \mathrm{C}$ [17], los cuales son superiores al obtenido para almidón de batata morada y amarilla. Una mayor temperatura de gelatinización en almidones nativos, refleja una mayor estabilidad interna del gránulo de almidón, normalmente asociada a una mayor presencia de zonas semicristalinas $[1,27,28,29,30]$.

En virtud de que las propiedades funcionales mostradas por los almidones nativos de los tubérculos tropicales fueron en algunos casos mejores al tradicional almidón de maíz, pero similares a las reportadas para las mismas variedades cultivadas en otra parte del mundo, la importancia de este trabajo radica en revalorar aquellos cultivos, como los tubérculos tropicales (batata), que, por diversas razones, han sido poco estudiadas, pero que por sus componentes tienen un prometedor potencial de explotación. Particularmente, el elevado contenido de almidón presente y la gran versatilidad de propiedades fisicoquímicas y funcionales encontradas para este polisacárido, hacen posible la utilización de los recursos naturales de la región, con la finalidad de contar con alternativas propias que permitan evitar, en lo posible, la dependencia del exterior de estos ingredientes alimentarios.

\section{CONCLUSIONES}

En la caracterización fisicoquímica del almidón de batata morada y amarilla no se encontraron diferencias significativas según la prueba t-student ( $p>0,05)$ en cuanto al contenido de humedad $(10,95$ y $10,91 \%)$; cenizas $(0,32$ y $0,38 \%)$; fibra cruda $(0,43$ y $0,47 \%)$; proteína $(0,38$ y $0,34 \%)$; grasa $(0,46$ y $0,20 \%)$, carbohidratos totales $(87,35$ y $87,69 \%)$; calorías (355,99 Kcal/100 g almidón y 353,91 Kcal/100 g almidón)

El almidón obtenido de batata amarilla presentó mejor calidad con mayor contenido de almidón con respecto al almidón de batata morada, ya que obtuvo una baja solubilidad, alta absorción de agua y un alto poder de hinchamiento en su caracterización funcional. La temperatura de gelatinización de los almidones de batata morada $\left(76,67^{\circ} \mathrm{C}\right)$ y amarilla $\left(76,33^{\circ} \mathrm{C}\right)$, pueden ser usados en productos que requieran altas temperaturas, tales como los productos enlatados, alimentos para bebés, entre otros. 


\section{REFERENCIAS}

[1] ACOSTA-DELGADO, ANNIE-PAULINA; BLANCO-SANTANDER, CATHERINE. Obtención y caracterización de almidones nativos colombianos para su evaluación como posibles alternativas en la industria alimentaria [Tesis Ingeniero de Alimentos]. Cartagena (Colombia): Universidad de Cartagena, 2013. 273 p.

[2] LESE, VILIAMU; HOLLAND, ELISABETH; WAIRIU, MORGAN; HAVEA, ROBIN; PATOLO, SOANE; NISHI, MINORU; HOPONOA, TANIELA; BOURKE, MICHAEL; DEAN, ANNIKA; WAQAINABETE, LOGOTONU. Facing food security risks: The rise and rise of the sweet potato in the Pacific Islands. Journal of Global Food Security, v. 18, 2018, p. 48-56. https://doi.org/10.1016/j.gfs.2018.07.004

[3] WANG, LIN; ZHAO, YING; ZHOU, QING; LUO, CHUN-LI; DENG, AI-PING; ZHANG, ZI-CHENG; ZHANG, JIU-LIANG. Characterization and hepatoprotective activity of anthocyanins from purple sweet potato (Ipomoea batatas L. cultivar Eshu No. 8). Journal of Food and Drug Analysis, v. 25, n. 3, 2016, p. 607-618. https://doi.org/10.1016/j.jfda.2016.10.009

[4] KOLAWOLE, FAUSAT; AKINWANDE, BOLANLE; ADE-OMOWAYE, BEATRICE. Physicochemical properties of novel cookies produced from orange-fleshed sweet potato cookies enriched with sclerotium of edible mushroom (Pleurotus tuberregium). Journal of the Saudi Society of Agricultural Sciences, v. 19, n. 2, 2020, p. 174-178. https://doi.org/10.1016/j.jssas.2018.09.001

[5] SOLARTE-MONTUFAR, JUAN G.; DIAZ-MURANGA, ANDERSON E.; OSORIO-MORA OSWALDO; MEJIA-ESPAÑA, DIEGO F. Propiedades reológicas y funcionales del almidón procedente de tres variedades de papa criolla. Información Tecnológica, v. 30, n. 6, 2019, p. 35-44. http://dx.doi.org/10.4067/S0718-07642019000600035

[6] GRANADOS, CLEMENTE; GUZMÁN, LUIS-ENRIQUE; ACEVEDO, DIOFANOR; DÍAZ, MARÍA; HERRERA, ANA. Propiedades funcionales del almidón de Sagu (Maranta arundinacea). Revista Biotecnología en el Sector Agropecuario y Agroindustrial, v. 12, n. 2, 2014, p. 90-96.

[7] VELÁSQUEZ-HERRERA, JULIAN-DAVID; LUCAS-AGUIRRE, JUAN-CARLOS; QUINTERO-CASTAÑO, VÍCTOR-DUMAR. Physical-chemical characteristic determination of potato (Solanum phureja Juz y Bukasov) starch. Acta Agronómica, v. 66, n. 3, 2017, p 323-330. https://doi.org/10.15446/acag.v66n3.52419

[8] AMERICAN SOCIETY FOR TESTING AND MATERIALS (AOAC). Official methods of analysis. 16th ed. Association of Official Analytical Chemists. Washington D.C. (USA): 1990.

[9] ANDERSON, R.A.; CONWAY, H.F.; PHEISER, V.F.; GRIFFIN, L. Gelatinization of corn grits by roll and extrusion cooking. Cereal Science Today, v. 14, 1969, p. 4-12. https://doi.org/10.1002/star.19700220408

[10] GRACE M.R. Elaboración de la yuca. Roma (Italia): Organización de las Naciones Unidas para la Agricultura y la Alimentación (FAO), 1977 116, p.

[11] MANZANILLAS-ROJAS, LISBETH-ADELA. Evaluación de las propiedades fisicoquímicas y funcionales de féculas de tres variedades de camote (Ipomoea batata) para aplicaciones alimentarias [Tesis Ingeniería de Alimentos]. Ambato (Ecuador): Universidad Técnica de Ambato, 2018, 168 p.

[12] ABEGUNDE, OLUWASEYI-KEMI; MU, TAI-HUA; CHEN, JING-WANG; DENG, FU-MING. Physicochemical characterization of sweet potato starches populrarly used in Chinese starch industry. Food Hydrocolloids, v. 33, n. 1, 2013, p. 169-177.

http://dx.doi.org/10.1016/j.foodhyd.2013.03.005

[13] GONZÁLEZ, A., OLGUÍN, N., LOAYZA, E.; SEVERICH, E. Almidón Nativo y Modificado. Obtención, cuantificación, modificación y usos [En línea)]. 2014. Disponible: http://www.dui.uagrm.edu.bo/Informacion/ InvestigacionesCHI/6-chi-2014.pdf. [Acceso: 29 de enero de 2019].

[14] MEDINA-GARCÍA, LUÍS. Obtención de maltodextrinas por vía enzimática a partir de almidón de camote (Ipomoea batatas) [Tesis Maestro en Ciencias en Producción Agrícola Sustentable]. Jiquilpan (México): Instituto Politécnico Nacional, 2013. 
[15] ALVIS, ARMANDO; VÉLEZ, CARLOS A.; VILLADA, HÉCTOR S.; RADA-MENDOZA, MAITE. Análisis Físico-Químico y Morfológico de Almidones de Ñame, Yuca y Papa y Determinación de la Viscosidad de las Pastas. Información Tecnológica, v. 19, n. 1, 2008, p.1928. http://dx.doi.org/10.4067/S0718-07642008000100004

[16] LEIVA-LÓPEZ, CORALIA-MERCEDES; OBANDO-PÉREZ, RAYZA-SOLANGE. Extracción de almidón a partir de variedades de papa cultivadas en Nicaragua [Tesis Ingeniero Químico]. Managua (Nicaragua): Universidad Nacional de Ingeniería, 2014, 60 p.

[17] SALCEDO, JAIRO-GUADALUPE; GARCÍA-MOGOLLÓN, CARLOS; SALCEDO-HERNÁNDEZ, DAVID. Propiedades funcionales de almidones de ñame (Dioscorea alata). Biotecnología en el sector agropecuario y agroindustrial, v. 16, n. 2, 2018, p. 99-107. http://dx.doi.org/10.18684/bsaa.v16n2.1170

[18] ACUÑA-PINTO, HAROLD-MAURICIO. Extracción, caracterización y aplicación de almidón de ñame variedad blanco (Dioscórea trífida) originario de la región amazónica colombiana para la elaboración de productos horneados [Tesis Especialización en Ciencia y Tecnología de Alimentos]. Bogotá (Colombia): Universidad Nacional de Colombia, 2012, 64 p.

[19] PANDI, J.; GLATZ, P.; FORDER, R.; AYALEW, W.; WARAMBOI, J.; CHOUSALKAR, K. The use of sweet potato (Ipomoea batatas, L) root as feed ingredient for broiler finisher rations in Papua New Guinea. Animal Feed Science and Technology, v. 14, 2016, p.1-11. https://doi.org/10.1016/j.anifeedsci.2016.01.011

[20] SHEKHAR, SHUBHENDU; MISHRA, DIVYA; BURAGOHAIN, ALAK-KUMAR; CHAKRABORTY, SUBHRA; CHAKRABORTY, NIRANJAN. Comparative analysis of phytochemicals and nutrient availability in two contrasting cultivars of sweet potato (Ipomoea batatas L.). Food Chemistry, v. 173, 2015, p. 957-965. https://doi.org/10.1016/j.foodchem.2014.09.172

[21] TORTOE, CHARLES; AKONOR, P.T.; KOCH, KRISTINE; MENZEL, CAROLIN. Physicochemical and functional properties of flour from twelve varieties of Ghanaian sweet potatoes. International Food Research Journal, v. 24, n. 6, 2017, p. 2549-2556.

[22] ARISTIZÁBAL, JOHANNA; SÁNCHEZ, TERESA; MEJÍA-LORIO, DANILO. Guía técnica para producción y análisis de almidón de yuca. Boletín de servicios agrícolas de la FAO, 163. Roma (Italia): Organización de las Naciones Unidas para la Agricultura y la Aminentación (FAO), 2007, p. 66-74.

[23] NARAYANA-MOORTHY, NUBRAMONY. Physicochemical and Functional Properties of Tropical Tuber Starches: A Review. Journal Starch - Starke, v. 54, n. 12, 2002, p. 559-592. https://doi.org/10.1002/1521-379X

[24] ZHU, FAN. Isolation, composition, structure, properties, modifications, and uses of yam starches. Coomprehensive review in food sciencie and food safety, v. 14, 2015, p. 257-386. https://doi.org/10.1111/1541-4337.12134

[25] MARTÍNEZ, P.; MÁLAGA, A.; BETALLELUZ, I.; IBARZ, A.; VELEZMORO, C. Caracterización funcional de almidones nativos obtenidos de papas (Solanum phureja) nativas peruanas. Revista Scientia Agropecuaria, v. 6, n. 4, 2015, p. 291-301. 10.17268/sci.agropecu.2015.04.06

[26] CERON-LASSO, MARÍA; ALZATE ARBELAEZ, ANDRÉS F.; ROJANO, BENJAMÍN A.; ÑUSTEZ-LÓPEZ, CARLOS E. Composición Fisicoquimica y Propiedades Antioxidantes de genótipos nativos de papa criolla (Solanum tuberosum grupo phureja). Información Tecnológica, v. 29, n. 3, 2018, p. 205-216. http://dx.doi.org/10.4067/S071807642018000300205

[27] IESE, VILIAMU; HOLLAND, ELISABETH; WAIRIU, MORGAN; HAVEA, ROBIN; PATOLO, SOANE; NISHI, MINORU; HOPONOA, TANIELA; BOURKE, MICHAEL; DEAN, ANNIKA; WAQAINABETE, LOGOTONU. Facing food security risks: The rise and rise of the sweet potato in the Pacific Islands. Global Food Security, v. 18, 2018, p. 48-56. https://doi.org/10.1016/j.gfs.2018.07.004 
[28] LOW, J.; BALL, A.M.; MAGEZI, S.; NJOKU, R.; MWANGA, M.; ANDRADE, M.; TOMLINS, K.; DOVE, R.; VAN MOURIK, R.D. Sweet potato development and delivery in sub-Saharan África. African Scholary Science, v. 17, n. 02, 2017, p. 11955-11972. 10.18697/ajfand.78.HarvestPlus07

[29] LEBOT, VINCENT. Rapid quantitative determination of maltose and total sugars in sweet potato (Ipomoea batatas L. [Lam.]) varieties using HPTLC. Journal of Food Science and Technology, v. 54, n. 3, 2017, p. 718-726. https://doi.org/10.1007/s13197-017-2510-2

[30] NGOMA, KHUTHADZO; MASHAU, MPHO; SILUNGWE, HENRY. Physicochemical and Functional Properties of Chemically Pretreated Ndou Sweet Potato Flour. International Journal of Food Science, 2019. https://doi.org/10.1155/2019/4158213 